\title{
Dynamic aspects for interislet synchronization of oscillatory insulin secretions
}

\author{
NAN-KUANG YAO, LIANG-WEY CHANG, BONIFACE JUISIANG LIN, AND TE-SON KUO \\ Department of Electrical Engineering, Center for Biomedical Engineering, and Graduate Institute \\ of Clinical Medicine, National Taiwan University, Taiwan, Republic of China
}

\begin{abstract}
Yao, Nan-Kuang, Liang-Wey Chang, Boniface Juisiang Lin, and Te-Son Kuo. Dynamic aspects for interislet synchronization of oscillatory insulin secretions. Am. J. Physiol. 272 (Endocrinol. Metab. 35): E981-E988, 1997.-How are the oscillatory insulin secretions from numerous islets synchronized to result in an identifiable oscillation? We postulated that a sudden increase in glucose concentration could best account for the interislet synchronization. The perifusion with two parallel chambers each containing 100 islets from the same rat was performed. The glucose concentrations of two chambers were simultaneously increased from 100 to 300 $\mathrm{mg} / \mathrm{dl}$ in step function to examine the synchronizing efficacy. Synchrony and regularity of insulin oscillation were evaluated by cross-correlation and/or power spectral analysis. Although the insulin had been in stable oscillation, we found that the synchrony between two chambers and the regularity of each chamber were still significantly improved after a sudden increase in glucose level. However, the improved synchrony and regularity were transient. They gradually slid toward a less rigorous condition in a 15 -h long-term perifusion. We suggested that the interislet synchronization of oscillatory insulin secretions could be improved by a sudden increase in glucose level. The insulin pulses were therefore enhanced to present their physiological effects.
\end{abstract}

synchrony; regularity; cross-correlation; spectral analysis

PANCREATIC ISLETS SECRETE insulin in an oscillatory fashion. Clearly identifiable oscillations with periods of 10-20 min were shown in experiments using intact animals $(13,21)$, perfused pancreata $(29)$, perifused isolated islets $(4,10,22)$, and adenomatous human $\mathrm{B}$ cells (11). The site of the pacemaker has now been narrowed down to the individual $\mathrm{B}$ cells. In addition, oscillations of $\mathrm{CO}_{2}$ production, cytoplasmic $\mathrm{Ca}^{2+}$, ATPto-ADP ratio, and lactate within the $\mathrm{B}$ cell have been described $(9,12,15)$. Correlation of these synchronous cycles with those observed for insulin secretion leads to a proposal that the oscillatory glycolysis within each B cell is probably the originator of the oscillatory rhythm. Therefore a set of new questions arises: how do the insulin secretions from thousands of B cells in a single islet and from millions of islets in pancreas coordinate sufficiently to result in a clearly identifiable oscillation? In the clusters of $\mathrm{B}$ cells, a digital fura 2 fluorescence image analysis indicates that the synchrony among the clusters of $\mathrm{B}$ cells can be achieved by rapid propagation of cytoplasmic $\mathrm{Ca}^{2+}(17)$. In a single islet, the analysis had been extended to confirm that the oscillations of $\mathrm{Ca}^{2+}$ concentrations occur synchronously across the whole islet tissue (26). Recently, the single pancreatic mouse islets were superfused to allow concomitant recordings of cytoplasmic $\mathrm{Ca}^{2+}$ and insulin release (2). It was found that the release of the insulin from a single islet indeed was synchronized with the $\mathrm{Ca}^{2+}$ oscillation. The intraislet synchronizing mechanism is therefore ascribed to a highly coordinated $\mathrm{Ca}^{2+}$ signaling system. In groups of islets, Berman et al. (5) proposed a mathematical model to examine the degree of synchrony required to obtain the oscillatory insulin patterns. They suggested a moderate degree of asynchrony between islets could best account for the pattern of insulin oscillation observed. However, the model of Berman et al. was based on a series of critical assumptions. Secretory events of all islets were assumed to be discrete bursts. The quantities and firing intervals of insulin releases for all islets were also assumed to be the same only with very small variation. It is not clear that the model still is applicable in the face of considerable biological uncertainties after a long-term operation. Nevertheless, the foregoing observations indicate that the oscillatory characteristics not only among groups of B cells but also among groups of islets are determined collectively. This phenomenon might account for the great variation of insulin oscillatory periods among different experimental designs. Because of the overlap by scattered insulin rhythms, an increase of islet number might accompany an increase of periods. It was reported that individual rat islets released insulin in 2- to 3 -min pulses $(2,3)$, but 10 - to 20 -min pulses were found in groups of isolated islets or perfused pancreata $(4,10,22,29)$. On the other hand, this phenomenon also implies that there should be more room for improving the synchrony of insulin oscillation. Their physiological effect (23) might therefore be enhanced with a shorter period and greater amplitude.

We postulated that a sudden increase in glucose concentration was the supplementary mechanism in synchronizing the insulin oscillations from groups of islets. A perifusion experiment was therefore designed with two parallel chambers containing groups of islets from the same rat. The system especially improved the accurate control in quantity and timing of the alteration in glucose level to practically examine the synchronizing efficacy. It was confirmed that although the insulin had been in stable oscillation, both thcir synchrony and regularity still were significantly improved after a sudden increase in glucose level. In addition, in a 15-h long-term perifusion, the synchrony and regularity improved by glucose increase were transient. They gradually slid toward a less rigorous condition similar to that before glucose alteration.

\section{MATERIALS AND METHODS}

Isolation of rat islets. Islets were isolated by use of the collagenase technique of Gotoh et al. (14). Male Wistar rats (NTU, Taipei, ROC) were anesthetized and $10 \mathrm{ml}$ of collage- 


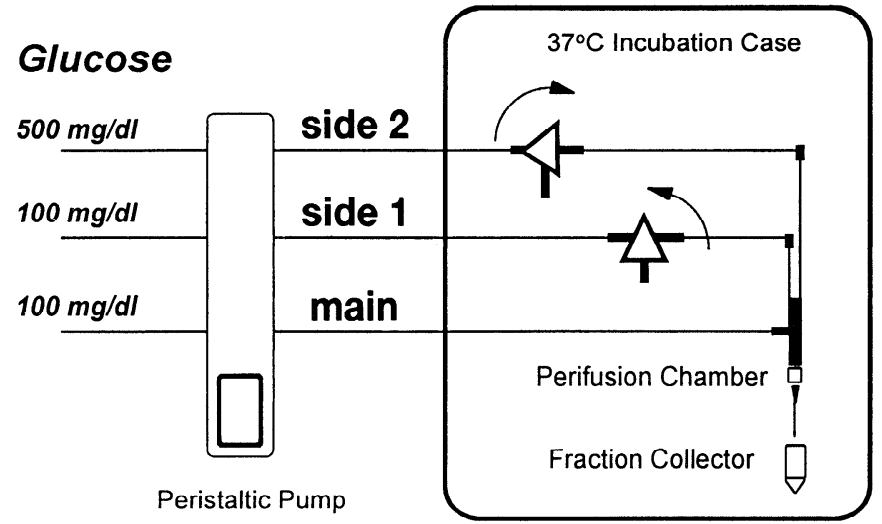

Fig. 1. Schematic representation of perifusion system. Tube arrangement was adopted from Watanabe and Orth (30) with some modifications. To begin glucose alteration, two 3-way stopcocks were turned simultaneously in opposite directions. Media in 2 side lines were immediately exchanged (side 1 to side 2 ). Two identical sets of tubes and chambers were operated simultaneously.

nase solution were injected into the common bile duct to distend the pancreas. It was excised and placed into a $37^{\circ} \mathrm{C}$ water bath for digestion. After a scries of washing steps, the islets were separated from other tissue fragments by centrifugation on a discontinuous Ficoll (Sigma, St. Louis, MO) gradient in Hanks' balanced salt solution with specific gravities of 1.080 and 1.110 . About 500 clean islets were obtained and cultured in RPMI 1640 medium containing $100 \mathrm{mg} / \mathrm{dl}$ glucose (GIBCO/BRL, supplemented with $0.2 \%$ sodium bicarbonate, $1.5 \% N$-2-hydroxyethylpiperazine- $N^{\prime}$-2-ethanesulfonic acid, $10 \%$ newborn calf serum, $0.2 \%$ penicillin-streptomycin, and $0.1 \%$ amphotericin $\mathrm{B}$ ) at $37^{\circ} \mathrm{C}$, in a $\mathrm{CO}_{2}$ incubator for $24 \mathrm{~h}$ to ensure the complete recovery of islets.

Perifusion system. In all experiments, two chambers ( $\alpha$ and $b$ ) containing groups of islets from the same rat were perifused simultaneously ( $n=6$ pairs, expts $1-6$ ). About 100 similar size islets were hand picked under the microscope and placed into each chamber, which was composed of a barrel of 1-ml plastic syringe ('Terumo, Tokyo, Japan), and two layers of nylon net with $10-\mu \mathrm{m}$ pore (Pharmacia, Uppsala, Sweden) were placed in the bottom. To control exactly the time of introduction of high-glucose medium into the chamber and to prevent the pressure disturbances, the method of tubing arrangement from Watanabe and Orth (30) was modified. Briefly, three parallel tubes (1 main line and 2 side lines for each chamber) were driven by a multichannel peristaltic pump at an individual flow rate of $180 \mu \mathrm{l} / \mathrm{min}$ (Fig. 1). Each of the side lines was connected with a three-way stopcock and a 21-gauge needle. A consistent flow of medium containing 100 $\mathrm{mg} / \mathrm{dl}$ glucose in the main line was always introduced into the chamber. Under basal perifusion condition, one side line (side 1 ), which also contained medium with glucose level of 100 $\mathrm{mg} / \mathrm{dl}$ (same as used for overnight culture, gassed with $95 \%$ $\mathrm{O}_{2}-5 \% \mathrm{CO}_{2}$ ), was inserted through the upper port of the chamber, and its three-way stopcock was opened. The chamber was therefore perifused with the basal glucose level of 100 $\mathrm{mg} / \mathrm{dl}$ at a flow rate of $360 \mu \mathrm{l} / \mathrm{min}$. Another side line (side 2), primed with medium containing $500 \mathrm{mg} / \mathrm{dl}$ glucose, was also inserted into the chamber until the tip of the needle reached close to the islets. To switch the glucose concentrations, two three-way stopcocks were turned in opposite directions simultaneously. Thereby the glucose level in the vicinity of islets was immediately switched to $300 \mathrm{mg} / \mathrm{dl}$ (an average of 100 and $500 \mathrm{mg} / \mathrm{dl}$ ), and the total flow rate still was $360 \mu \mathrm{l} / \mathrm{min}$. Because only a half-volume was disturbed and the total flow rate through the chamber was maintained throughout the operation, the significant pressure change was prevented. The system demonstrated a perfect step function of glucose alteration and nearly no time shift between the two chambers (Fig. 2). The temperature of the entire system was monitored and controlled by an electronic controller to exclude fluctuation. Variation was no more than $0.1^{\circ} \mathrm{C}$ over $300 \mathrm{~min}$ and demonstrated no periodicity.

Experimental protocols. Samples were collected in 3-min fractions. To equilibrate the islet conditions, the islets were perifused with the same medium as during overnight culture for $2 \mathrm{~h}$ before the start of sample collection. The islets were therefore considered to secrete insulin in a stable oscillation at $100 \mathrm{mg} / \mathrm{dl}$ glucose level. The glucose levels of two chambers in all experiments were simultaneously increased from 100 to $300 \mathrm{mg} / \mathrm{dl}$ in step function. In experiments 1-5, the switching point was at 90 min after sample collection started, and the duration was designed to obtain 90 samples $(90+180 \mathrm{~min})$. Experiment 6 was an independent long-term perifusion in which the switching point was at $180 \mathrm{~min}$, and the perfusion was prolonged to obtain 300 samples $(180+720 \mathrm{~min})$.

Radioimmunoassay. Insulin concentrations in the collected perifusate were determined by radioimmunoassay with ${ }^{125} \mathrm{I}$ labeled human insulin and human standard (19), employing an anti-insulin serum raised in guinea pig (a gift from Dr. C. H. Hsu, Jen-Ai Municipal Hospital, Taipei, ROC). Dextrancoated charcoal for insulin was used as a separation method. The intra- and interassay coefficients of variation were 4.5 and $7.2 \%$. The limit of sensitivity was $1.2 \mu \mathrm{U} / \mathrm{ml}(7.2 \mathrm{pM})$. All samples were measured in duplicates. The consistent trend of the signal profiles from the duplicate assays were further examined pulse by pulse to verify the real peaks or valleys of insulin oscillations.

Data analysis. The synchrony and regularity of insulin oscillation were characterized by cross-correlation (7) and the power spectral analysis (8). Cross-correlation is essentially a comparison process to determine the phase shift of signal that has been hidden in additive noise. The primary application of cross-correlation is the synchrony estimate between two signals. Synchrony between two signals was defined as if there were a significant peak corresponding to zero lag time in cross-correlogram. In addition, the regularity of the trace in a cross-correlogram also approximately reflects the regular-

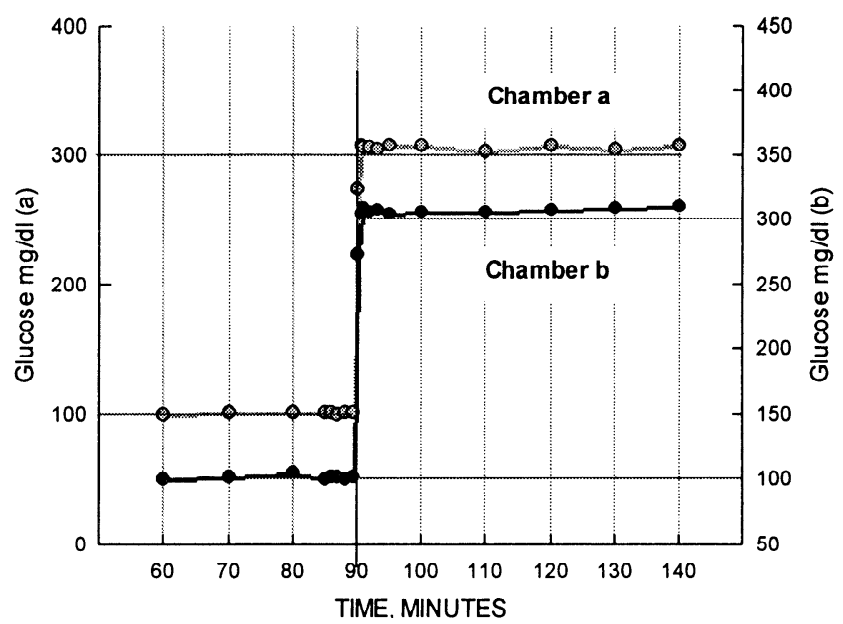

Fig. 2. Rapid response of perifusion system to a sudden alteration in glucose concentration. Perifusate from 2 parallel chambers ( $a$ and $b$ ) was immediately measured by a glucose analyzer (model 2300 STATPlus, Yellow Springs Instrument, Yellow Springs, OH). Sampling interval was reduced to $30 \mathrm{~s}$ near switching point. Note that scale for chamber $b$ (on right) was shifted. 


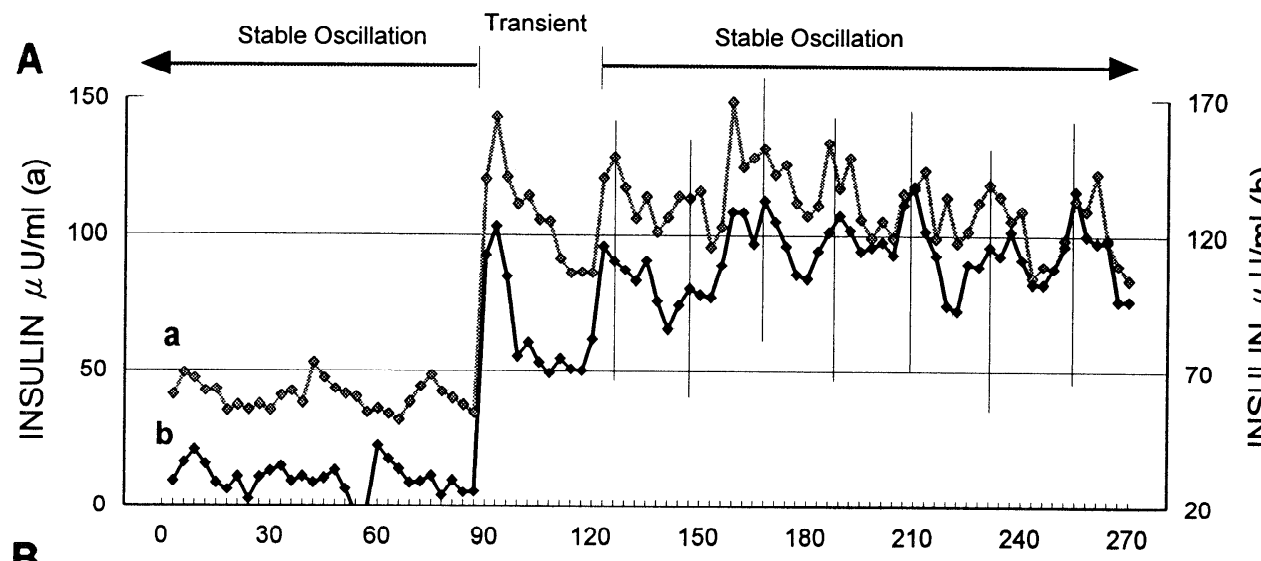

Fig. 3. A: oscillatory insulin sccretions by 2 groups of perifused islets (chambers $a$ and $b$ ) from a single rat (expt 1). Glucose levels in 2 parallel chambers were simultaneously changed from 100 to $300 \mathrm{mg} / \mathrm{dl}$ at 90 min. Period of time from 90 to $120 \mathrm{~min}$ was ignored in assessment of stable oscillation after switch. Note that insu-

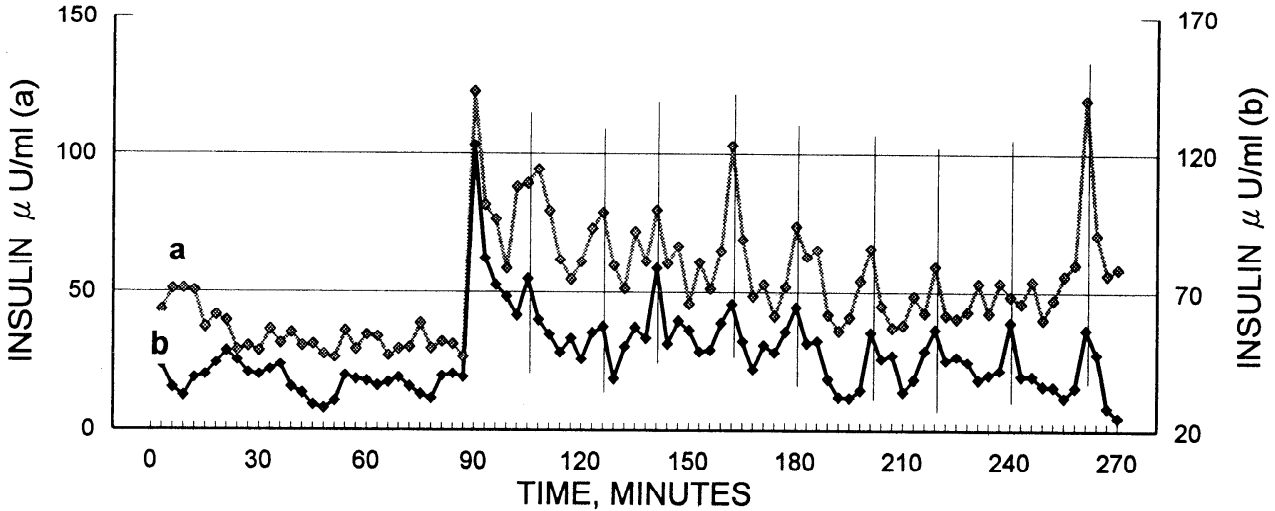
lin scale for chamber $b$ (on right) was shifted. $B$ : insulin oscillations in a repeat experiment with another rat (expt 2).

ity of two original signals similar to that of autocorrelation. We also evaluated regularity by power spectral analysis based on the Fourier transform. A plot of the spectral densities against periods provides a good visual display of the structure of the waveform and the power distributions among each period component. The duration from the glucose switching point to $30 \mathrm{~min}$ later was considered to include most of the transient state in response to glucose increase, i.e., firstphase insulin secretion. To avoid interference with the calculation, data in this duration were ignored. Two separate ranges at both sides were therefore defined as the stable oscillation before the switch and the stable oscillation after the switch (Fig. 3). In experiment 6, the perifusion was prolonged to $900 \mathrm{~min}$. Included were $180 \mathrm{~min}$ of stable oscillation before the switch defined as range 0 and a $690-\mathrm{min}$ $(900-180-30=690)$ stable oscillation after switch, in which five overlapping moving windows (60 data points, 180 min) were defined as ranges 1-5 (Fig. 6). The time series of data of every range were curve fitted with a third-order polynomial as a detrending function to exclude low-frequency bias. The cross-correlation between chambers $a$ and $b$ and power spectral analysis for each chamber was then performed range by range. Because the amplitudes of insulin oscillation would be enhanced when glucose level increased, correlation coefficients and spectral densities were normalized to compare the synchrony and regularity before and after the switch. The foregoing analyses were performed with Signal Processing Toolbox of MATLAB, IBM-PC version (Math Works, Natick, MA).

\section{RESULTS}

Figure $3 A$ shows the results of experiment 1 . Insulin in chambers $a$ and $b$ presented a very consistent dynamic response, sustained oscillations superimposed on the well-known first- and second-phase secretions. Figure $3 B$ shows the results of the same experiment with islets from another rat (expt 2). The dynamic behaviors in two parallel chambers were also very consistent in response to increased level of glucose. However, both the duration of the first phase and the period of subsequent oscillation in experiment 2 were significantly shorter than those of experiment 1 . Table 1 shows the duration of the first-phase secretion, from the switching point to the subsequent stable oscillation, in experiments 1-6. They varied among individual experiments but were similar for each pair of chambers. Synchrony betwecn paired chambers was further analyzed by cross-correlation (Figs. 4 and 7). The traces of cross-correlograms show bias at both sides corresponding to a large time shift. It was due to the finite length of the signals and did not interfere with estimates of synchrony or regularity. A significant peak indeed existed corresponding to zero lag time in the cross-correlograms after switching in all experiments.

Table 1. Duration of first-phase insulin secrelion

\begin{tabular}{ccc}
\hline \hline & \multicolumn{2}{c}{ Duration of 1st Phase, min } \\
\cline { 2 - 3 } Expt No. & Chamber $a$ & Chamber b \\
\hline 1 & 33 & 30 \\
2 & 12 & 15 \\
3 & 18 & 18 \\
4 & 18 & 21 \\
5 & 15 & 15 \\
6 & 15 & 15
\end{tabular}



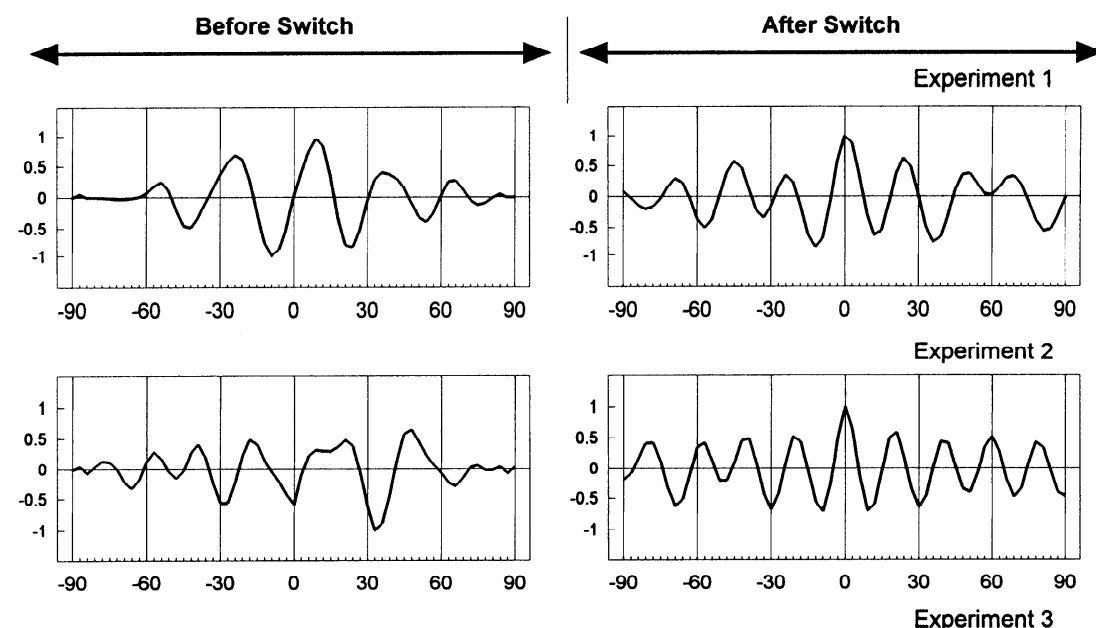

Fig. 4. Cross-correlation of stable insulin oscillation between 2 parallel chambers before and after switching glucose levels $(100$ to $300 \mathrm{mg} / \mathrm{dl})$ for 5 repeat experiments (expts 1-5). Correlation coefficients had been normalized for comparison. Synchrony was defined as if there were a significant peak corresponding to 0 lag time, and regularity was evaluated by regular cycles in cross-correlogram.
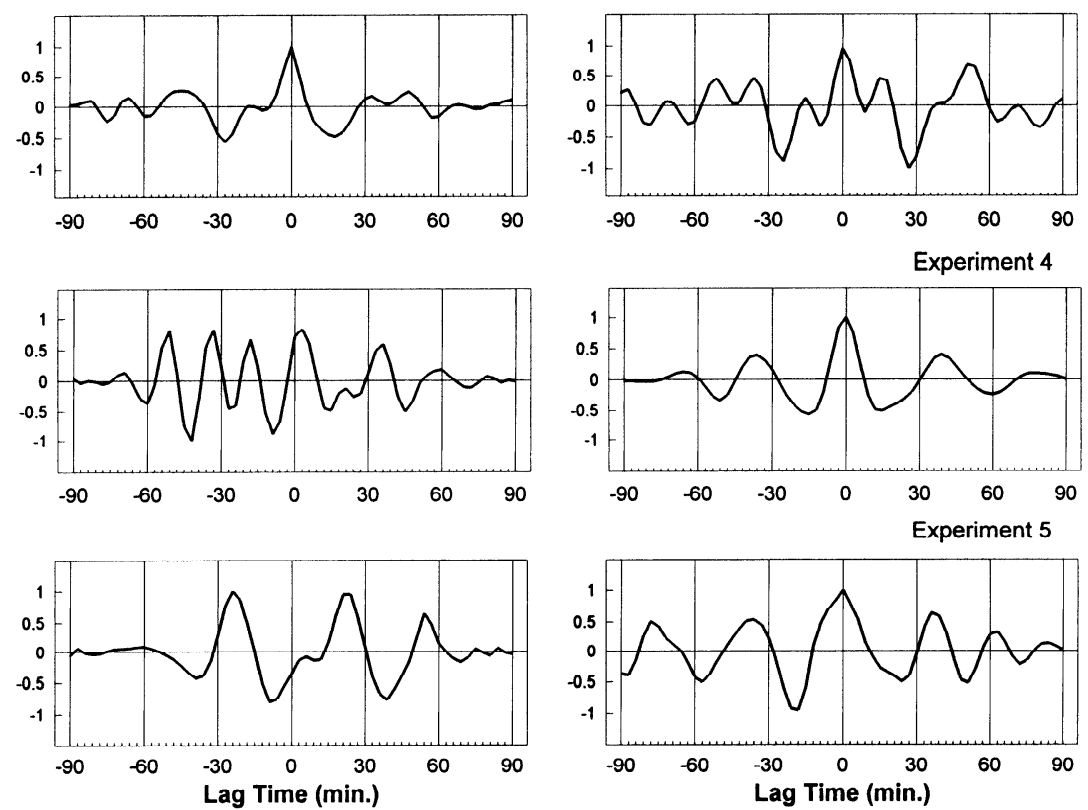

Except for experiment 3, they were improved from asynchronous oscillation. This implied that a high degree of synchrony had been achieved by a sudden increase in glucose concentration. In addition, regular cycles in cross-correlogram reflecting the regularity of two original signals were also identified in five experiments (expts 1,2, and 4-6) after switch. In experiments 2 and $4-6$, they were improved from irregular traces. Enhancement of regularity was also supported by the spectral analysis (Figs. 5 and 7). After glucose levels were switched, the dominant peaks for each pair of chambers had been converged. This implied that most power of the insulin oscillations from both chambers had been concentrated to an identical frequency. Like the duration of first-phase secretion, the spectral patterns after the switch were quite different among experiments but were similar for paired chambers in each experiment.

In experiment 6, the perifusion was extended to 900 min (15 h; Fig. 6$)$. The synchrony and regularity in the stable oscillations before the switch (range 0 ) and after the switch (ranges 1-5) were also analyzed range by range with power spectrum and cross-correlation (Fig.
7). In the spectral analysis, the dominant peaks of the two chambers converged after switching of the glucose level (range 1), but then gradually diverged thereafter (ranges 2-5). Moreover, the two spectral patterns of paired chambers were considerably similar in the initial two ranges after the switch (ranges 1 and 2), but then they became different in the later ranges (ranges $3-5)$. The synchrony analysis by cross-correlation also presented consistent results with experiments 1-5. However, a significant peak corresponding to zero lag time and the regular cycles in cross-correlogram were observed only in the first range after the switch (range 1).

\section{DISCUSSION}

Four possible factors affecting the interislet synchronizing process are neural network, $\mathrm{Ca}^{2+}$ propagation, temperature variation and change in fuel concentration. Because groups of isolated rat islets also secrete insulin in a clearly oscillating fashion in the absence of neural interconnection, there should be another mechanism in addition to neural regulation. Is it possible that 
Before Switch
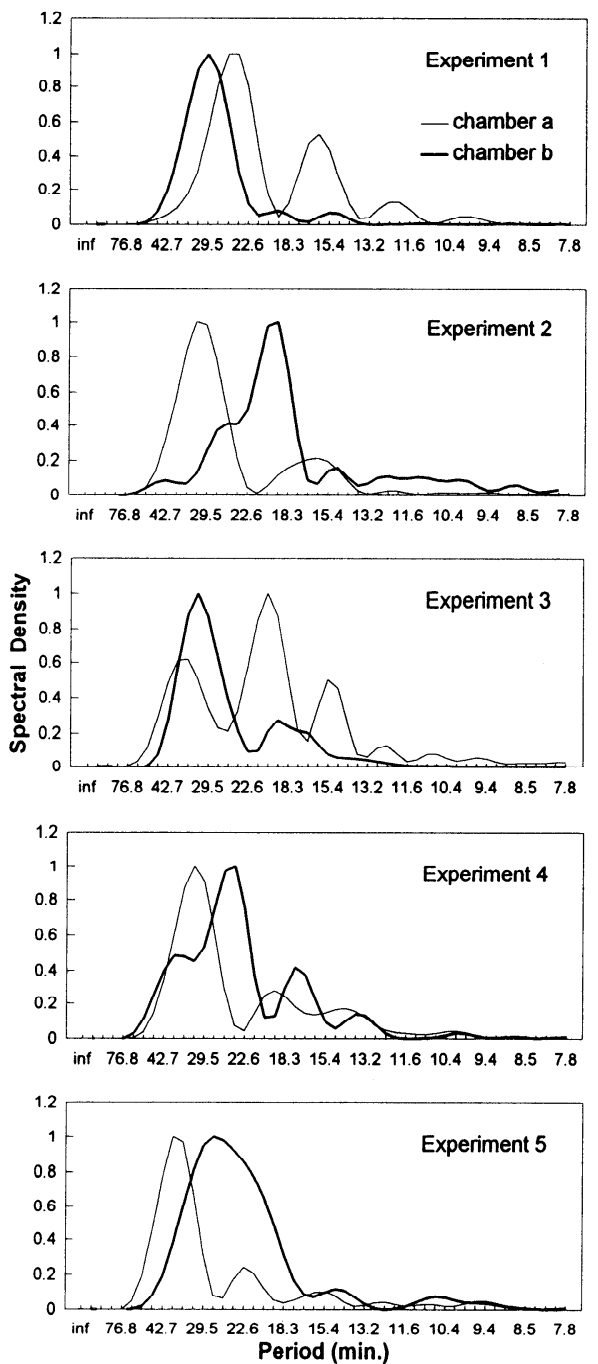

After Switch
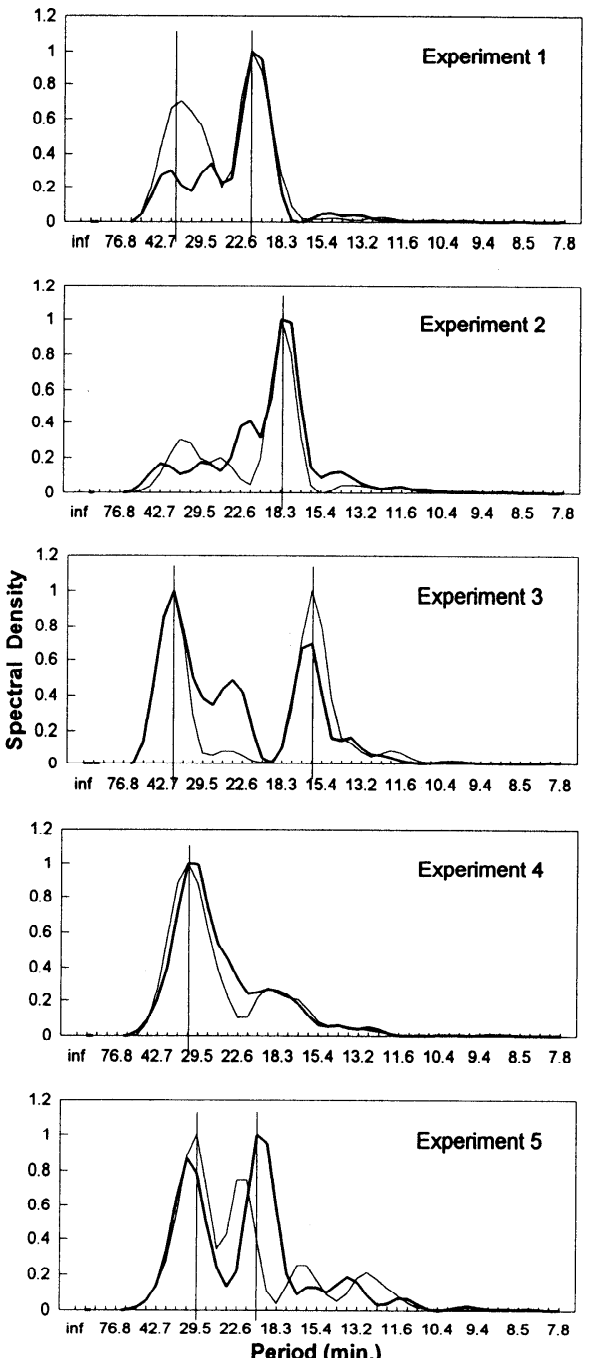

Fig. 5. Power spectral analyses of stable insulin oscillations before and after switching glucose levels (100 to $300 \mathrm{mg} / \mathrm{dl}$ ) for 5 repeat experiments (expts 1-5). Thin lines and dark bold lines represent chambers $a$ and $b$, respectively. Spectral density was normalized.
Fig. 6. Oscillatory insulin secretions by perifused islets from a single rat (expt 6 ). Glucose levels in 2 parallel chambers $(A$, chamber $a ; B$, chamber $b$ ) were simultaneously raised from 100 to $300 \mathrm{mg} / \mathrm{dl}$ at $180 \mathrm{~min}$. Perifusion after switch was extended to $900 \mathrm{~min}$, resulting in 690 min of stable oscillation, during which 5 overlapping moving windows were defined as ranges $1-5$. Initial $180 \mathrm{~min}$ was considered to be period of stable oscillation before switching and was defined as range 0 .

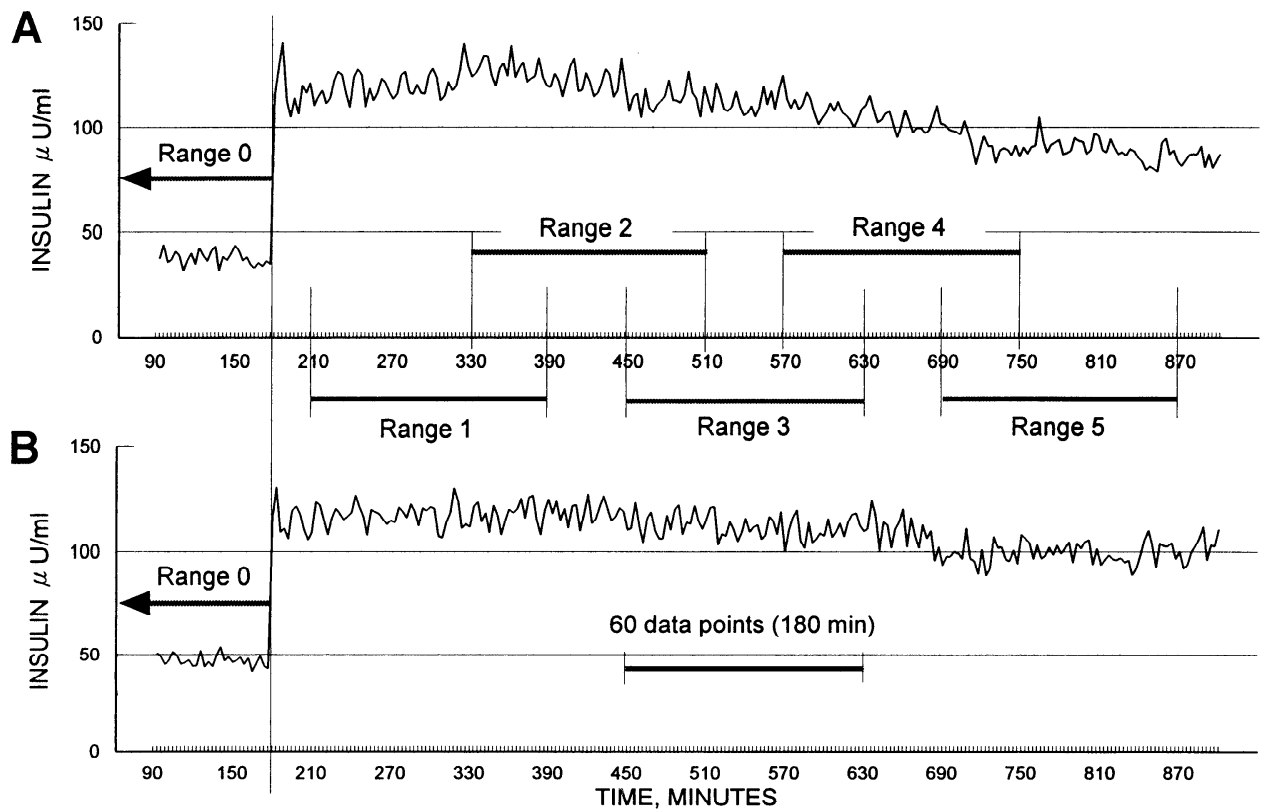



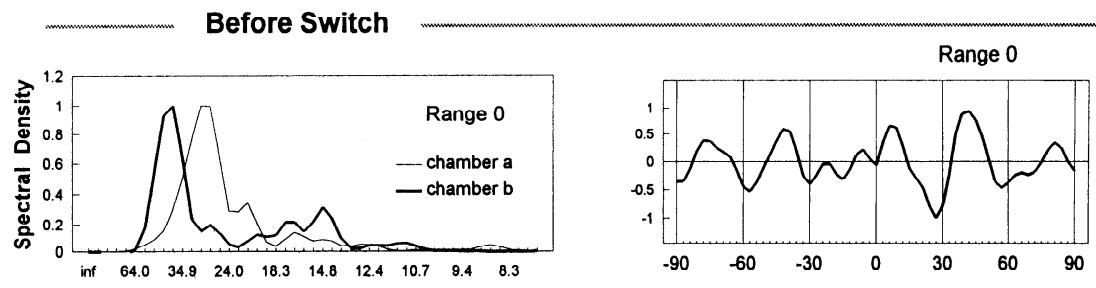

Fig. 7. Range-by-range power spectra (on left) and cross-correlation analyses (on right) of stable insulin oscillations before and after switching glucose levels for expt 6. Both spectral densities against periods and correlation coefficients against lag time were normalized.
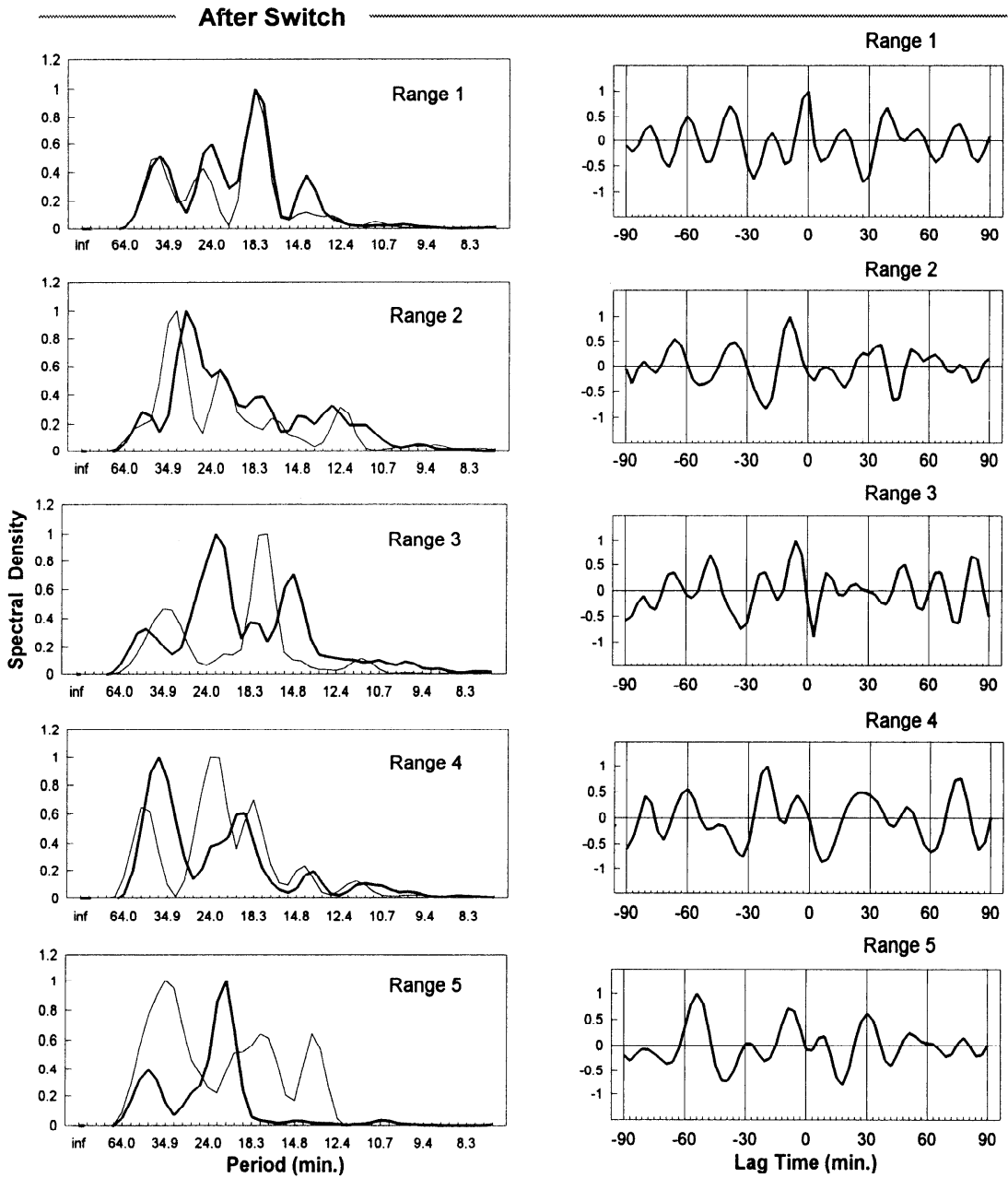

a widespread $\mathrm{Ca}^{2+}$ propagation, i.e., paracrine and/or endocrine flow of $\mathrm{Ca}^{2+}$, occurs in the pancreas? The fura 2 fluorescence image analysis indicates that the phase shift between oscillations occurring in different B cells in one single islet is $\sim 2 \mathrm{~s}(26)$. It is unlikely that millions of islets scattered in the widespread pancreas can be sufficiently coordinated to result in a clear insulin oscillation within a few minutes just by a paracrine flow of $\mathrm{Ca}^{2+}$. To facilitate the propagation of $\mathrm{Ca}^{2+}$ by the endocrine pancreas, there should be an efficient microvascular pathway from islet to islet. Recently, comparison of the results obtained from the anterograde vs. retrograde perfusion of the pancreas indicated the existence of the cellular perfusion sequence, $\mathrm{B} \rightarrow \mathrm{A} \rightarrow \mathrm{D}$, in the islets of various animals (25, 27,28 ). This finding leads to a corollary that most islets in the pancreas are connected in parallel. It is because any specific intraislet perfusion sequence, including $\mathrm{B} \rightarrow \mathrm{A} \rightarrow \mathrm{D}$, is hardly established if these islets are connected in series. The corollary is also supported by the reconstruction of islet microvasculature (6). At least in rats, the blood in most islets is introduced from arteries and then drained directly to veins without passing through any other islet. Thus, it is clear that the islet-to-islet endocrine pathway for $\mathrm{Ca}^{2+}$ is lacking in the pancreas. A third possible mechanism is the temperature variation. Hellman et al. (18) reported that oscillation of cytoplasmic $\mathrm{Ca}^{2+}$, reflecting the oscillation of insulin secretion, is inhibited when the temperature is above $42^{\circ} \mathrm{C}$ or below $30^{\circ} \mathrm{C}$. If insulin cycles can be synchronized by reinitiating the oscillation by temperature, the mechanism must operate under unphysiological temperature conditions (near 42 or $30^{\circ} \mathrm{C}$ ). Oscillatory temperature in normal range might make an impulsive periodicity of insulin. Yet, it is also hard to perform in vivo because the body temperature must permanently oscillate in synchronous cycles with insulin rhythms. The final possible 
mechanism is a sudden increase of fucl concentration, such as glucose and/or arginine. Arginine is a powerful amino acid stimulus of both insulin and glucagon secretions. However, the concomitantly released glucagon might disturb the insulin cycles, since the high level of glucagon will initiate the transformation of oscillatory $\mathrm{Ca}^{2+}$ concentration in B cells into a sustained increased level (15). Therefore arginine or arginine-stimulated glucagon might not be able to synchronize the scattered insulin rhythms by means of $\mathrm{Ca}^{2 \text { । }}$ oscillation. It was reported that the transition from the basal state to the oscillatory state of cytoplasmic $\mathrm{Ca}^{2+}$ is induced by raising the glucose concentration to $7 \mathrm{mM}$ $(126 \mathrm{mg} / \mathrm{dl})$ or above (15). This implies that glucose alteration in the normal physiological range is able to completely shut down or to initiate the insulin oscillation. This established the possibility of glucose as a good trigger to rally the scattered insulin rhythms from numerous islets.

However, this mechanism should start out with the following anatomic and dynamic premises.

Each islet should first receive signal of glucose increase at the same time. Because the blood flow only needs a few seconds to pass through the pancreas, the blood glucose signal could be considered to arrive at every islet in pancreas at the same time. The problem is that the other hormones that are also secreted by islet, such as glucagon, somatostatin, and even insulin itself, have been identified as influencing insulin secretion (1, 24, 20). Each islet therefore must receive the glucose signal first to avoid any interference from hormones released by other islets. As discussed previously, most islets in the pancreas are connected in parallel by the blood circulation system. This observation suggests not only that islets are independent of each other but also that each of them should contact the glucose signal first.

Each islet should be consistent in response time to a sudden increase in glucose level. Two dynamic characteristics have to be considered: the time it takes the islet to receive the glucose signal and begin secreting insulin (firing time) and the length of time from the initial peak to the beginning of a new stable oscillation (duration of 1 st phase). In fact, insulin response to a sudden increase in glucose is strong and immediate. It had been well documented and also confirmed by our results. In addition, our results reveal that although the duration of the first-phase insulin secretion was varied among different rats, it was similar for paired chambers. Thus, for the individual rat, this premise was satisfied.

In this study, two groups of islets from the same rat were perifused in the absence of any contact pathway. The glucose levels of two groups of islets were simultaneously increased to examine the synchronizing efficacy. All the methodological pitfalls, such as temperature variation or pressure disturbance resulting in an artificial synchronization, were prevented. We found that a high degree of synchrony between two chambers was achieved by a sudden increase in glucose concentrations. This can readily be seen through identification of a significant peak corresponding to zero lag time in cross-correlograms after switching of the glucose levels in all experiments. Most of them were improved from asynchronous oscillation, since the similar phenomena were not found before switching. The peak-to-peak distances, especially among the peaks near zero lag time, would reflect the periods of two original signals. However, it is meaningful only if the two signals are synchronous and the trace in cross-correlogram is regular. In our results, regular cycles in cross-correlogram were also identified after switching of the glucose levels, which made it possible to estimate the periods of the two insulin cycles. Apparently, the periods derived by cross-correlation coincided with those observed from the original data (Fig. 4 vs. 3). On the other hand, after switching of the glucose level, the dominant peaks in the spectrums of chambers $a$ and $b$ converged and the spectral patterns of the two chambers became unified. This implied that most power of both insulin oscillations from two groups of islets became concentrated to an identical frequency; i.e., insulin secretions from chambers $a$ and $b$ presented a pair of regular oscillations with a consistent period.

In experiment 6 , a high degree of synchrony between the two chambers was transient because the significant peak at zero lag time in cross-correlogram was found only in range 1. Meanwhile, the spectral patterns of both chambers were also unified only in range 1 . They gradually diverged in the later ranges (ranges $2-5$ ) and finally presented individual spectral patterns. This implied that both groups of the islets had developed individual oscillatory characteristics after prolonged culture in the absence of any contact pathway between the two chambers. We think that the loss of synchrony between the two chambers was due to the difference between their individual oscillatory characteristics and that the loss of synchrony in one chamber resulted in its specific oscillatory characteristic. In any given chamber, some biological uncertainties, such as the variations in the oscillatory glycolysis and in the flow of cytoplasmic $\mathrm{Ca}^{2+}$, exist in the secretory events among islets. Trivial variations of the secretory events could become so accumulated after long-term perifusion as to render insulin rhythms scattered among islets, but the insulin rhythms did not scatter in a completely random fashion. First, identifiable insulin oscillations were observed throughout the perifusion, and the dominant peaks in spectrum of both chambers were always found even in the final range of the perifusion (range 5 in expt 6 ). Second, the insulin rhythms indeed slid toward a less rigorous condition similar to that before the switch (range 5 vs. range 0, Fig. 7) or, as Berman et al. (5) suggested, a moderate degree of asynchrony. Therefore insulin secretions from the islets in one chamber finally presented individual oscillatory characteristics by the overlap of moderate degree of scattered insulin rhythms. The unified spectral pattern in range 1 might be closer to the natural oscillatory characteristics of islets. This is because the overlap of scattered insulin rhythms had been reduced by the high degree of synchrony in this range. It is somewhat surprising that the spectral 
patterns under synchronized condition were so much alike for islets from a single rat but so different among different rats. Whether this is due to the different living history of rats or just to different degree of damage in the isolation procedure remains to be investigated.

Conclusion. Our results established the possibility that scattered insulin rhythms from numerous islets in pancreas could be synchronized by alterations in glucose levels in the arteries. Although insulin secretions had been in a stable oscillation, there still was room for improvement in their synchrony and regularity by a sudden increase in glucose concentrations. However, the improved synchrony and regularity were transient. They would gradually slide toward a less rigorous condition after prolonged culture if lacking an effective regulatory mechanism. We suggest that, after a sudden increase in glucose concentrations, the oscillations of insulin would be improved in synchrony and regularity. Each time the blood glucose level increased, the oscillation of insulin would be automatically synchronized. The insulin pulses were therefore enhanced to present their physiological effects.

The authors thank the following colleagues for expert technical assistance: Chao-Ying Kuo, Chung-Huei Hsu, Ging-Shing Justin Won, Huey-Peir Wu, Sheng-Huei Tang, and Yi-Fan Yang.

This work was supported by the National Science Council ('Taiwan) under Grant NSC 85-2331-B-002-219 M08 and the National Health Research Institute (Taiwan) under Grant DOH 85-HR-310.

Address for reprint requests: L.-W. Chang, Center for Biomedical Engineering, College of Medicine, National Taiwan University, No. 1, Sec. 1, Jen-Ai Road, Taipei, Taiwan, Republic of China.

Received 17 October 1996; accepted in final form 13 February 1997.

\section{REFERENCES}

1. Alberti, K. G. M. M., N. J. Christensen, S. E. Christensen, A. P. Hansen, J. Iversen, K. Lundbaek, K. Seyer-Hansen, and H. Ørskov. Inhibition of insulin secretion by somatostatin. Lancet 8: 1299-1301, 1973.

2. Bergsten, P., E. Grapengiesser, E. Gylfe, A. Tengholm, and B. Hellman. Synchronous oscillations of cytoplasmic $\mathrm{Ca}^{2+}$ and insulin release in glucose-stimulated pancreatic islets. J. Biol. Chem. 269: 8749-8753, 1994

3. Bergsten, P., and B. Hellman. Glucose-induced amplitude regulation of pulsatile insulin secretion from individual pancreatic islets. Diabetes 42: 670-674, 1993.

4. Bergstrom, R. W., W. Y. Fujimoto, D. C. Teller, and C. De Haën. Oscillatory insulin secretion in perifused isolated rat islets. Am. J. Physiol. 257 (Endocrinol. Metab. 20): E479-E485, 198.9.

5. Berman, N., H. F. Chou, A. Berman, and E. Ipp. A mathematical model of oscillatory insulin secretion. Am. J. Physiol. 264 (Regulatory Integrative Comp. Physiol. 33): R839-R851, 1993.

6. Bonner-Weir, S., and L. Orci. New perspectives on the microvasculature of the islets of Langerhans in the rat. Diabetes 31: 883-889, 1982.

7. Challis, R. E., and R. I. Kitney. Biomedical signal processing. 1. Time-domain methods. Med. Biol. Eng. Comput. 28: 509-524, 1990.

8. Challis, R. E., and R. I. Kitney. Biomedical signal processing. 3. The power spectrum and coherence function. Med. Biol. Eng. Comput. 29: 225-241, 1991.

9. Chou, H. F., N. Berman, and E. Ipp. Oscillations of lactate released from islets of Langerhans: evidence for oscillatory glycolysis in $\beta$-cells. Am. J. Physiol. 262 (Endocrinol. Metab. 25): E800-E805, 1992.

10. Chou, H. F., and E. Ipp. Pulsatile insulin secretion in isolated rat islets. Diabetes 39: 112-117, 1990 .

11. Chou, H. F., E. Ipp, R. R. Bowsher, N. Berman, C. Ezrin, and S. Griffiths. Sustained pulsatile insulin secretion from adenoma- tous human $\beta$-cells. Synchronous cycling of insulin, C-peptide, and proinsulin. Diabetes 40: 1453-1458, 1991

12. Corkey, B. E., K. Tornheim, J. T. Deeney, M. C. Glennon, J. C. Parker, F. M. Matschinsky, N. B. Ruderman, and M. Prentki. Linked oscillations of free $\mathrm{Ca}^{2+}$ and ATP/ADP ratio in permeabilized RINm5F insulinoma cells supplemented with a glycolyzing cell-free muscle extract. J. Biol. Chem. 263: 42544258, 1988 .

13. Goodner, C. J., B. C. Walike, D. J. Koerker, J. W. Ensinck, A. C. Brown, E. W. Chideckel, J. Palmer, and L. Kalnasy. Insulin, glucagon, and glucose exhibit synchronous, sustained oscillations in fasting monkeys. Science 195: 177-179, 1977.

14. Gotoh, M., T. Maki, S. Satomi, J. Porter, S. Bonner-Weir, C. J. O'Hara, and A. P. Monaco. Reproducible high yield of rat islets by stationary in vitro digestion following pancreatic ductal or portal venous collagenase injection. Transplantation 43: 725$730,1987$.

15. Grapengiesser, E., E. Gylfe, and B. Hellman. Glucose sensing of individual pancreatic $\beta$-cells involves transitions between steady-state and oscillatory cytoplasmic $\mathrm{Ca}^{2+}$. Cell Calcium 13: 219-226, 1992.

16. Grapengiesser, E., E. Gylfe, and B. Hellman. Glucoseinduced oscillations of cytoplasmic $\mathrm{Ca}^{2+}$ in the pancreatic $\beta$-cclls. Biochem. Biophys. Res. Commun. 151: 1299-1304, 1988.

17. Gylfe, E., E. Grapengiesser, and B. Hellman. Propagation of cytoplasmic $\mathrm{Ca}^{2+}$ oscillations in clusters of pancreatic $\beta$-cells exposed to glucose. Cell Calcium 12: 229-240, 1991.

18. Hellman, B., E. Gylfe, E. Grapengiesser, U. Panten, C. Schwanstecher, and C. Heipel. Glucose induces temperaturedependent oscillations of cytoplasmic $\mathrm{Ca}^{2+}$ in single pancreatic $\beta$-cells related to their electrical activity. Cell Calcium 11: 413-418, 1990.

19. Hsu, C. H., T. H. Hong, K. W. Yin, T. K. Tang, S. C. Wang, S. T. Chen, L. S. Lee, and T. H. Chang. Purification of radioiodinated human insulin by high performance liquid chromatography for a sensitive radioimmunoassay. J. Formosan Med. Assoc. 91: 9-14, 1992.

20. Iversen, J., and D. W. Miles. Evidence for a feedback inhibition of insulin on insulin secretion in the isolated, perfused canine pancreas. Diabetes 20: 1-9, 1971.

21. Jaspan, J. B., E. Lever, K. S. Polonsky, and E. Van Cauter. In vivo pulsatility of pancreatic islet peptides. Am. J. Physiol. 251 (Endocrinol. Metab. 14): E215-E226, 1986.

22. Marchetti, P., D. W. Scharp, M. Mclear, R. Gingerich, E. Finke, B. Olack, C. Swanson, R. Giannarelli, R. Navalesi, and P. E. Lacy. Pulsatile insulin secretion from isolated human pancreatic islets. Diabetes 43: 827-830, 1994.

23. Matthews, D. R., B. A. Naylor, R. G. Jones, G. M. Ward, and R. C. Turner. Pulsatile insulin has greater hypoglycemic effect than continuous delivery. Diabetes 32: 617-621, 1983.

24. Samols, E., G. Marri, and V. Marks. Promotion of insulin secretion by glucagon. Lancet 28: 415-416, 1965.

25. Samols, E., J. I. Stagner, R. B. L. Ewart, and V. Marks. The order of islet microvascular cellular perfusion is $\mathrm{B} \rightarrow \mathrm{A} \rightarrow \mathrm{D}$ in the perfused rat pancreas. .J. Clin. Invest. 82: 350-353, 1988.

26. Santos, R. M., L. M. Rosario, A. Nadal, J. Garcia-Sancho, B. Soria, and M. Valdeolmillos. Widespread synchronous $\left[\mathrm{Ca}^{2+}\right]_{i}$ oscillations due to bursting electrical activity in single pancreatic islets. Pflügers Arch. 418: 417-422, 1991.

27. Stagner, J. I., E. Samols, D. J. Koerker, and C. J. Goodner. Perfusion with anti-insulin gamma globulin indicates a B to A to $D$ cellular perfusion sequence in the pancreas of the rhesus monkey, Macaca mulatta. Pancreas 7: 26-29, 1992

28. Stagner, J. I., E. Samols, and S. Bonner-Weir. $\beta \rightarrow \alpha \rightarrow \delta$ pancreatic islet cellular perfusion in dogs. Diabetes 37: 17151721, 1988.

29. Stagner, J. I., E. Samols, and G. C. Weir. Sustained oscillations of insulin, glucagon, and somatostatin from the isolated canine pancreas during exposure to a constant glucose concentration. J. Clin. Invest. 65: 939-942, 1980.

30. Watanabe, T., and D. N. Orth. Detailed kinetic analysis of adrenocorticotropin secretion by dispersed rat anterior pituitary cells in a microperifusion system: effects of ovine corticotropinreleasing factor and arginine vasopressin. Endocrinology 121: $1133-1145,1987$. 